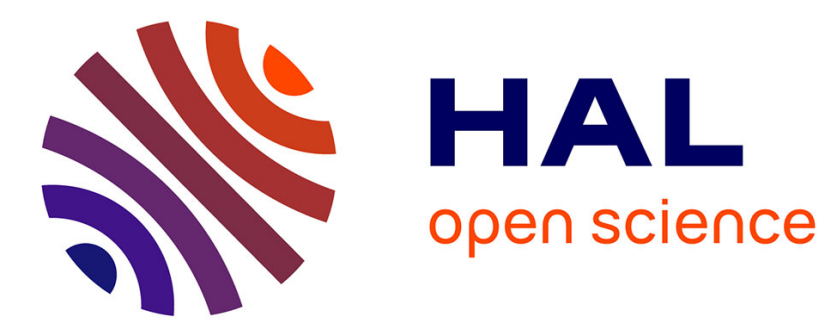

\title{
Effect of a noisy driving field on a bistable polariton system
}

H. Abbaspour, Gregory Sallen, Stephane Trebaol, F. Morier-Genoud, Marcia T. Portella-Oberli, B. Deveaud

\section{- To cite this version:}

H. Abbaspour, Gregory Sallen, Stephane Trebaol, F. Morier-Genoud, Marcia T. Portella-Oberli, et al.. Effect of a noisy driving field on a bistable polariton system. Physical Review B: Condensed Matter and Materials Physics (1998-2015), 2015, 92 (16), pp.165303. 10.1103/PhysRevB.92.165303 . hal-01211576

\section{HAL Id: hal-01211576 https://hal.science/hal-01211576}

Submitted on 12 Oct 2015

HAL is a multi-disciplinary open access archive for the deposit and dissemination of scientific research documents, whether they are published or not. The documents may come from teaching and research institutions in France or abroad, or from public or private research centers.
L'archive ouverte pluridisciplinaire HAL, est destinée au dépôt et à la diffusion de documents scientifiques de niveau recherche, publiés ou non, émanant des établissements d'enseignement et de recherche français ou étrangers, des laboratoires publics ou privés. 


\title{
Effect of a noisy driving field on a bistable polariton system
}

\author{
H. Abbaspour, ${ }^{1, *}$ G. Sallen, ${ }^{1}$ S. Trebaol, ${ }^{2}$ F. Morier-Genoud, ${ }^{1}$ M. T. Portella-Oberli, ${ }^{1}$ and B. Deveaud ${ }^{1}$ \\ ${ }^{1}$ Laboratory of Quantum Optoelectronics, École Polytechnique Fédérale de Lausanne, CH-1015 Lausanne, Switzerland \\ ${ }^{2}$ UMR FOTON, CNRS, Université de Rennes 1, Enssat, F22305 Lannion, France
}

(Received 20 April 2015; revised manuscript received 30 August 2015; published 2 October 2015)

\begin{abstract}
We report on the effect of noise on the characteristics of the bistable polariton emission system. The present experiment provides a time-resolved access to the polariton emission intensity. We evidence the noise-induced transitions between the two stable states of the bistable polaritons. It is shown that the external noise specifications, intensity and correlation time, can efficiently modify the polariton Kramers time and residence time. We find that there is a threshold noise strength that provokes the collapse of the hysteresis loop. The experimental results are reproduced by numerical simulations using Gross-Pitaevskii equation driven by a stochastic excitation.
\end{abstract}

DOI: 10.1103/PhysRevB.92.165303

PACS number(s): 78.20.Ls, 42.65.-k, 76.50.+g

\section{INTRODUCTION}

Optical bistable systems share the characteristic to present two possible output steady states for the same input light intensity. This is observed as a hysteresis cycle in the plot of the output light intensity versus input light intensity. Optical bistability was proposed by [1-3] and since then observed in many different systems such as cavity lasers [4-7], atomic systems [7,8], semiconductors [9-11], and microcavity polaritons [12,13]. Bistability is commonly used for device applications [3,14-16], particularly in polariton systems as spin switch and optical memory [17,18], optical transistor [19], laser [20], and logic functions [21]. The fidelity of the devices depends on the stability of the system.

Nonlinearity and optical feedback constitute the basis of optical bistability. In a bistable regime, instabilities can play an important role in the output signal. In fact, nonlinearity coupled with feedback fluctuations may induce transitions between the two stable states. Therefore, a noisier bistable system can give stochastic fluctuations in the output power. The characteristic time scale for these fluctuations is given by the transition probability defined by the residence time [22,23] and Kramers time [24], which depend on the noise strength and correlation time [23]. Moreover, bistable systems in the presence of fluctuations may appear as a discriminator instead of a hysteresis loop. In order to get insight into the actual bistable behavior of the system, which is related to the temporal variation of the population, we need to investigate the time behavior of the output intensity. Likewise, this time-resolved photon statistics might well reveal the genuine instability of the system, which should be considered in the realization of devices. Then, conversely, bistable optical systems can also provide an opportunity to investigate the fluctuations related to the nonlinear system.

Microcavity exciton polaritons are quasiparticles that arise from the strong coupling between excitons and photons [25]. They show a nonlinear behavior due to polariton interactions coming from their excitonic content, and their properties can be accessed through the emitted photons. Indeed, polariton repulsive interactions produce a positive nonlinear feedback mechanism when using a detuned exciting beam, which

\footnotetext{
*ahadis@phys.ethz.ch
}

can be observed as a hysteresis cycle in the output light intensity $[12,13]$. The relative energy positions of the input laser and of the polariton state play an important role in the polariton energy shift, which leads to optical bistability regimes with different hysteresis widths.

If the exciting laser is free of noise, at a given excitation power polaritons will stay either in the lower or in the upper branch of the hysteresis where they are stable. Otherwise, in the presence of noise and when the input laser power is within the bistable cycle, polaritons can escape from one of the stable branches to the other. The average time that the system stays in one bistable state before escaping to the other is defined as residence time. Then, with a certain amount of noise strength, polaritons undergo noise-assisted transitions between the steady states, and with a characteristic residence time they jump randomly up and down due to their coupling to the fluctuations. These fluctuations can originate from either external sources (the driving laser) or internal sources as a consequence of polariton with phonon and reservoir interactions. It has been theoretically predicted that the width of the measured hysteresis depends on the strength of the noise [23]. Therefore, time-resolved analysis of the output emission is crucial to shed light on the effect of noise in bistable polariton system.

In this paper, we report on the characteristics of polariton bistable systems by applying a controllable noise strength. We evaluate the effect of noise on the characteristics of a bistable polariton system. We show that the presence of noise induces a reduction of the width of the hysteresis cycle within the residence time scale. Moreover, the results reveal the noise threshold for which the hysteresis loop collapses into a discriminator. The investigation is pursued by the study of the emitted photon statistics through time-resolved measurements. We give evidence that the polariton emission intensity fluctuates between the lower and the upper states of the polariton bistability due to noise-induced transitions. We determine the lower- and upper-state residence times as a function of noise and excitation power. We determine the power for which the residence times of both branches are the same, which identifies the polariton Kramers time and we measure the Kramers times as a function of noise strength. Numerical simulations using Gross-Pitaevskii equation driven by a stochastic excitation reproduce very well the experimental results.

The paper is organized as follows. In Sec. II, we describe the sample and the experiments. Section III reports on 
the bistability measurements and time-resolved experimental studies. Section IV is dedicated to the theoretical model based on Gross-Pitaevskii equation driven by stochastic excitation. We give our conclusions in Sec. V.

\section{EXPERIMENTAL METHOD}

The sample is a single $8-\mathrm{nm} \mathrm{In}_{0.04} \mathrm{Ga}_{0.96} \mathrm{As}$ quantum well embedded in a GaAs $\lambda$ microcavity with AlAs/GaAs distributed Bragg mirrors [26]. The Rabi splitting is $3.5 \mathrm{meV}$ at zero detuning. Polaritons are confined in a patterned 3 - $\mu \mathrm{m}$-diameter mesa engineered on top of the spacer layer. The experiments are performed with the sample held at a temperature of $4 \mathrm{~K}$ and at a cavity-exciton detuning of $\delta=$ $-1.15 \mathrm{meV}$. The linewidth of the zero-dimensional polariton ground state is $\gamma=70 \mu \mathrm{eV}$, evidencing that the unavoidable disorder in the system only has static consequences that do not affect the present measurements. This makes it possible to perform experiments with the single ground-state level properly isolated from the other states. We plot in Fig. 1(b) the mesa emission spectrum showing the ground and the first excited state with energy separation of $1.01 \mathrm{meV}$. We use a linearly polarized single-mode $\mathrm{cw}$ Ti:sapphire laser with $10 \mathrm{MHz}$ linewidth and $2 \%$ noise intensity standard deviation. Using the noise eater, we reduce this value to $0.02 \%$. Then the laser beam passes through an electro-optic modulator, which imprints on the dc laser power a controllable noise intensity with Gaussian profile [Fig. 1(c)] and a 1.1-MHz bandwidth which is indeed much slower than the polariton intrinsic dynamics. We give the noise strengths as normalized standard deviation compared to the bistability width $\Delta \mathrm{B}$ measured without any additional noise on the laser. We vary the laser power, with a resolution equal to $1 \mu \mathrm{W}$, through a motorized rotating half-wave plate and a Glan polarizer. The sample is then excited at normal incidence with the laser circularly polarized to ensure polariton repulsive interactions and therefore polariton energy blueshift [27]. The laser has a spot diameter of $20 \mu \mathrm{m}$ and is of $\Delta=0.4 \mathrm{meV}$ detuned above the ground state, consequently satisfying the bistability condition $\Delta>\sqrt{3} \gamma$ [12]. The excitation and transmitted light are detected with 20-MHz-bandwidth photodiodes 2 and 3, respectively. The time-resolved measurements are performed with a 60-MHz-bandwidth oscilloscope.

\section{EXPERIMENTAL RESULTS}

\section{A. Effect of noise on polariton bistability}

In what follows, we present both experimental and theoretical results; details on the theory will only be given in Sec. IV. First we measure the polariton emission intensity as function

(a)

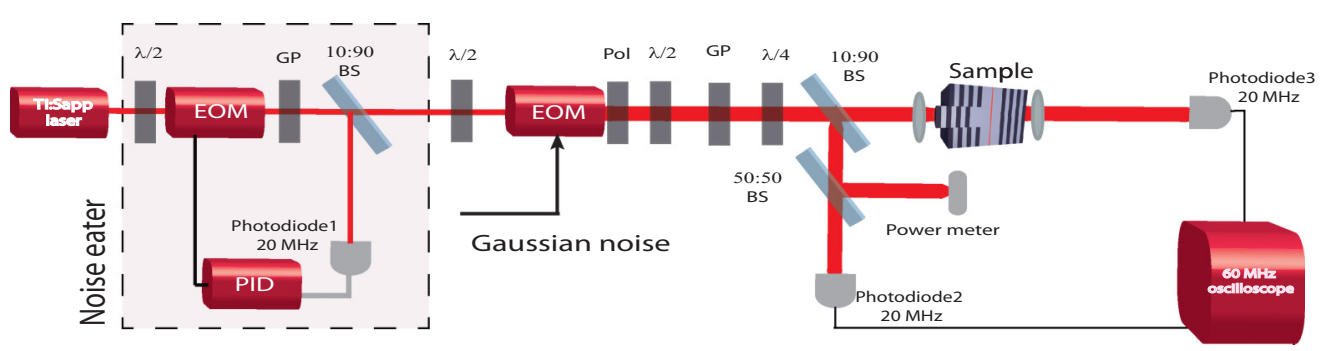

(b)

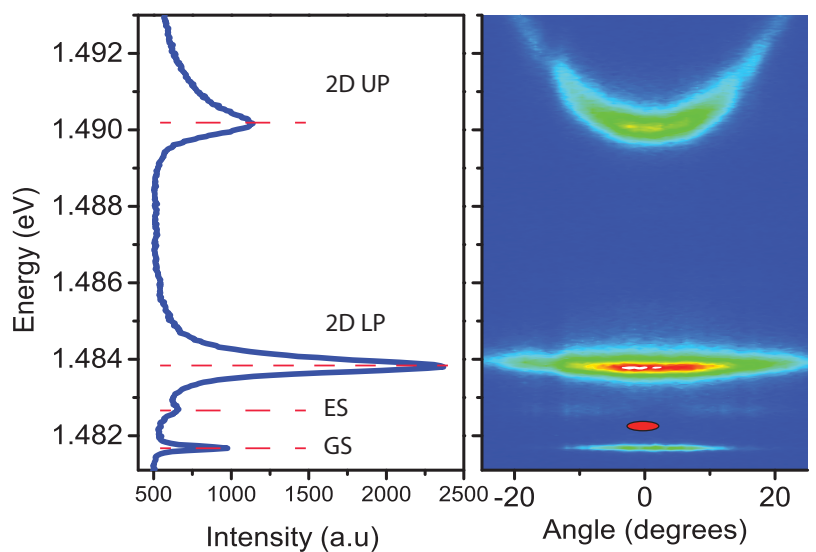

(c)

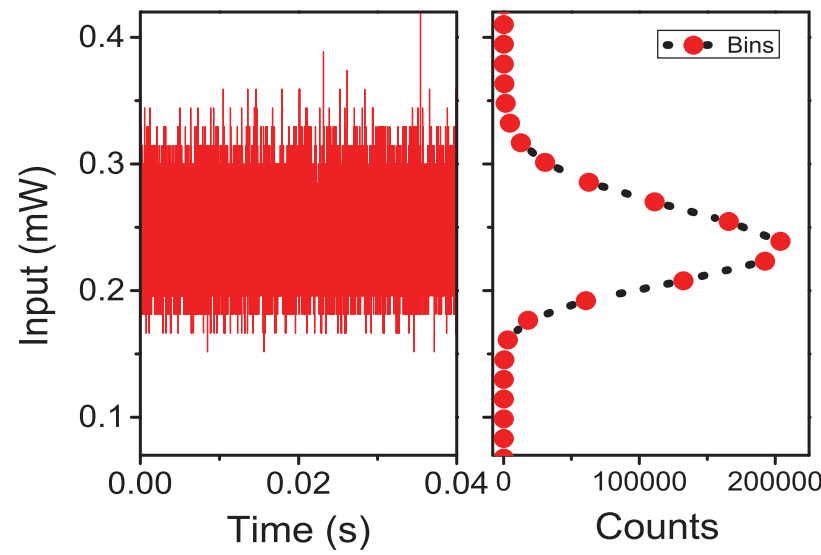

FIG. 1. (Color online) (a) Experimental setup. The microcavity sample is excited using a cw Ti:sapphire laser. Noise eater: the laser noise intensity is reduced to $0.02 \%$ through the first electro-optic modulator and a feedback loop (photodiode 1 and PID). The second EOM imprints a 1.1-MHz Gaussian noise on the laser intensity. The exciting and transmitted powers are detected with two fast photodiodes. BS, Pol, GP, $\lambda / 2$, and $\lambda / 4$ represent beam splitter, polarizer, Glan polarizer, half-wave plate, and quarter-wave plate, respectively. (b) Emission spectrum (left) and reciprocal space image (right) of the luminescence of the $3-\mu \mathrm{m}$ mesa. The energy position of the excitation laser is indicated by the red spot. 2D UP (LP), GS, and ES refer to the two-dimensional upper (lower) polariton branch, the ground state, and the excited state of the zero-dimensional polariton resonance, respectively. (c) Optical noise imprinted on the laser intensity (left) and Gaussian profile of the noise (right). 

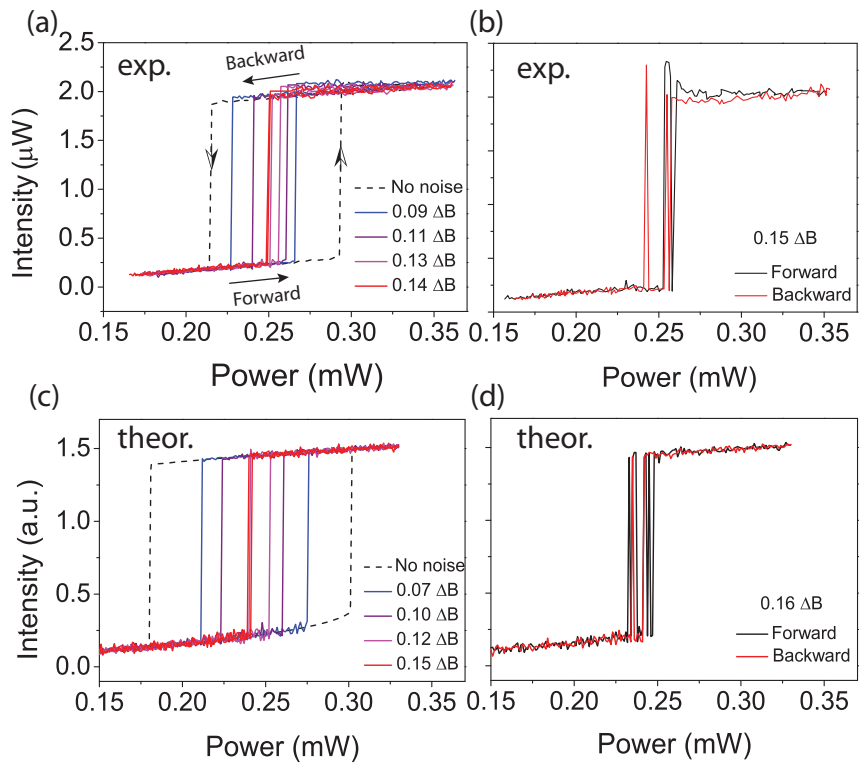

FIG. 2. (Color online) Zero-dimensional polariton bistability affected by the external Gaussian noise. (a) The initial experimental polariton bistability (dashed line) and polariton bistabilities for different amounts of the laser noise: $D=0.095,0.11,0.13$, and 0.14 $\Delta \mathrm{B}$. Fluctuations on the applied laser intensity make the polariton bistability unstable. We measure the collapse of the optical hysteresis. (b) For larger amounts of the laser noise $(D=0.15 \Delta \mathrm{B})$ we start to observe transition between two states. (c) The initial theoretical polariton bistability (dashed line) and polariton bistabilities for different amounts of the laser noise: $D=0.07,0.10,0.12$, and 0.15 $\Delta \mathrm{B}$. (d) For a larger amount of the laser noise $(D=0.16 \Delta \mathrm{B})$ we start to observe transitions between two states. The parameters used for the GPE are $\gamma_{p}=0.08 \mathrm{meV}, \alpha_{1}=0.34 \mathrm{meV}$, and $\Delta=0.4 \mathrm{meV}$. $\Delta \mathrm{B}$ is the width of the hysteresis without applying any noise.

of input laser power. The variation of the input power in the forward and backward directions builds the bistability curve in the output power. We repeat the measurements for a wide range of applied noise strengths. In Fig. 2(a), we display a set of bistability curves obtained for different amounts of applied noise. The bistability measured without any additional noise on the laser power (black dashed line) shows a hysteresis cycle width of $\Delta \mathrm{B}=68 \pm 8 \mu \mathrm{W}$. The uncertainty on the bistability width originates from unavoidable experimental mechanical noise and also internal noise sources. We observe that the increase in the noise-strength results in a decrease in the hysteresis width. Small amounts of noise perturb the emission behavior close to the bistability thresholds, inducing a single transition to the other branch in which the system stays stable. This reduces the width of the hysteresis cycle. Upon a certain amount of noise (here about $0.14 \Delta \mathrm{B}$ ) the hysteresis is screened and the system behaves as an optical discriminator at input power $0.245 \pm 0.008 \mathrm{~mW}$. Notice that, for larger noise strengths, some instability appears in the hysteresis cycle and random jumps occur between lower and upper branches [Fig. 2(b)]. This evidences that although the system behaves always as a bistable system, the intensity noise brings instability for device applications.

In Fig. 3(a), we plot the measured hysteresis width as a function of the applied noise strength, and in Fig. 3(b) we (a)
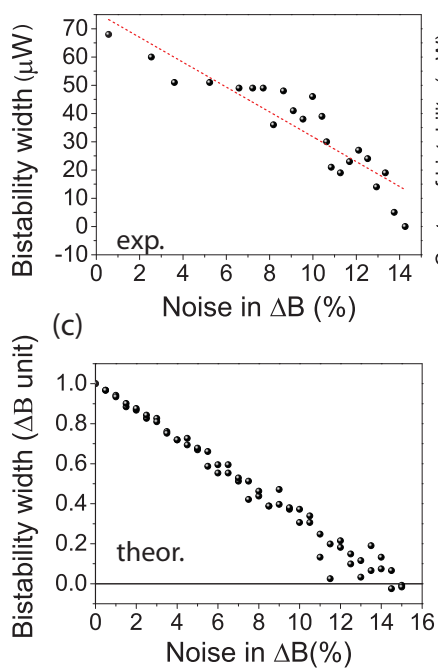

(b)

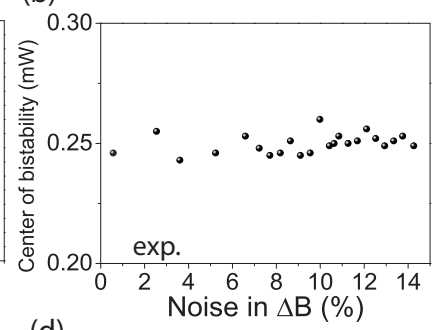

(d)

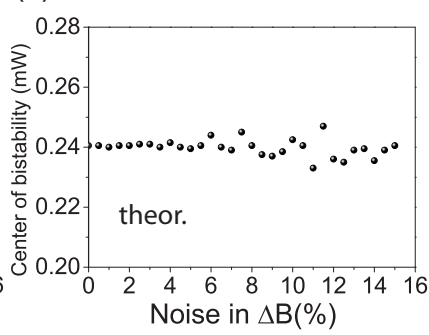

FIG. 3. (Color online) Characteristics of polariton bistability for different amounts of external noise. (a) Experimental bistability width versus intensity of laser fluctuations. When increasing the noise power, the bistability width decreases and, for $D=0.14$ $\Delta \mathrm{B}$, it vanishes. (b) For different amplitudes of the laser intensity fluctuations, the middle point of each hysteresis loop fluctuates around $0.245 \mathrm{~mW}$. (c) Theoretical bistability width (normalized to the maximum theoretical $\Delta \mathrm{B}$ ) versus noise strength. When increasing the noise power, the bistability width decreases and it vanishes for $D=0.15 \Delta \mathrm{B}$. (d) The middle point of each hysteresis loop fluctuates around $0.24 \mathrm{~mW}$. Unlike in the experiments, fluctuations become negligible at low noise.

display the input power corresponding to the middle position of the respective hysteresis loops. This result reveals a linear dependence of the bistability width with noise. In addition, the reduction of the hysteresis loop evolves symmetrically to collapse in the center position. By extrapolating the straight line we obtain the maximum width for the system free of any noise applied to the laser $(\Delta \mathrm{B} \approx 76 \mu \mathrm{W})$. It is worth mentioning that the real maximum bistability width can be even larger than this value. This difference originates from the minimal amount of noise which might be due to either external low-frequency mechanical or thermal vibrations or even intrinsic noise in the polariton system. Notice that such noise sources might be at the origin of the uncertainty in the experimental $\Delta \mathrm{B}$ and in the position of the center of the bistability loop at low applied noise.

To understand the dependence of the hysteresis cycle upon noise, it is very important to know how the bistable polariton system behaves. Note that in a bistable polariton system, the intensity of polariton emission is governed by both the polariton lifetime and the polariton-polariton interactions, which strongly depend on the polariton population. In addition, the emission intensity depends on the spectral overlap between the laser field and the polariton state. Within the hysteresis cycle, in the forward direction, we observe that the emission intensity in the lower branch increases linearly with the laser power [Fig. 2(a)]. This is due to the fact that the overlap between the laser line and the polariton state is very small. Therefore, the emission intensity increases simply due to the 
enhancement of the polariton population with laser power. However, this supply mechanism competes with the polariton lifetime loss mechanism. At a given threshold power, the feedback mechanism becomes dominant and polaritons reach a population at which interactions lock the polariton energy in resonance with the laser. This results in a better polariton coupling to the laser. This occurs at the upper power threshold of the hysteresis cycle, when the emission intensity jumps to the upper branch. In the backward direction, when decreasing the laser power, the emission remains in the upper branch until reaching the lower power threshold. This occurs at lower power because the polariton state is clamped to the laser and therefore both the polariton population and the nonlinearity strength are also clamped. At this lower threshold the losses due to polariton lifetime dominate the feedback mechanism, which reduces the polariton population. As a consequence, the nonlinear polariton interaction decreases and, accordingly, the polariton redshifts, which causes the loss of the polariton-laser locking. Therefore, the emission gets back to the low-intensity branch.

Furthermore, when introducing noise on the laser power, we directly induce fluctuations on the polariton population, which, due to nonlinearity, bring fluctuations on polariton energy. Therefore, the presence of noise in the system disturbs the competition between feedback and loss mechanisms. This appears to be more sensitive close to the upper and lower threshold powers when the equilibrium between both mechanisms is vulnerable. As a consequence, the fluctuations induce a transition from one to the other branch before attaining the threshold power, which causes the observed narrowing of the bistability width.

\section{B. Time-resolved experiments: Residence time and Kramers time}

In order to study the principle of noise-induced transitions, we perform a time-resolved analysis of the polariton emission. As mentioned before, when the laser intensity, including the noise, reaches either the lower or the upper threshold of bistability, the polariton population jumps to the corresponding stable state. Figure 4(a) shows the laser intensity as measured through photodiode 2 . The red dashed lines represent the lower and upper thresholds of optical bistability. Photodiode 3 allows us to measure synchronously the polariton intensity. When the laser intensity (including noise) reaches the lower threshold (points 1,3, and 5), polariton population jumps from the upper to the lower state, while when it reaches the upper threshold (points 2, 4, and 6), the polariton population jumps to the upper state [Fig. 4(b)]. This example clarifies the mechanism of polariton transition between well-defined stable states, which is based on reaching the nonlinear thresholds via laser fluctuations. To investigate the relation between the change of bistability cycle and this type of transition, we measure, at different input powers, the time behavior of the polariton emission intensity for the same experimental condition as Sec. III A for a wide range of noise strengths. In Figs. 5(a) to 5(d) we display the time streams of the polariton emission intensity recorded for different input laser powers at an applied noise strength of $0.30 \Delta \mathrm{B}$. We observe that at $0.24 \mathrm{~mW}$ polaritons jump between the two stable states with similar (a)

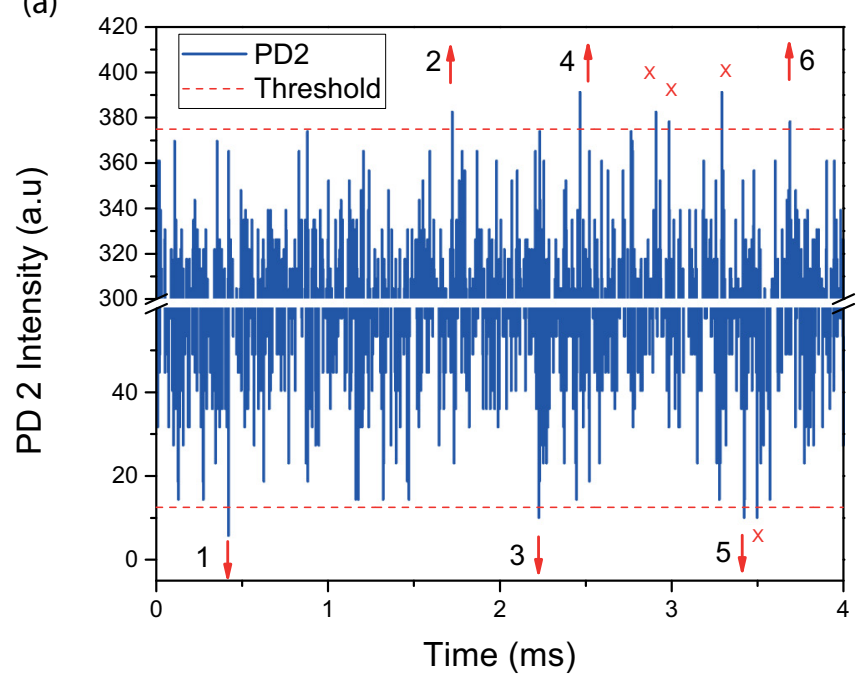

(b)

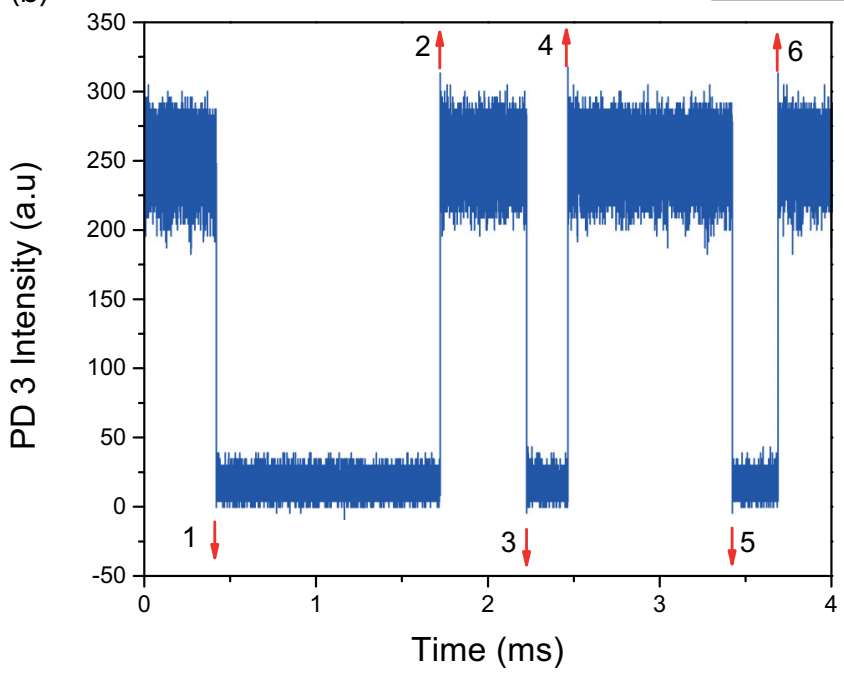

FIG. 4. (Color online) Principle of noise-induced transitions. (a) Time trace of the input laser intensity with added noise (with Gaussian profile). The red dashed lines represent the lower and upper thresholds of the optical bistability. (b) Synchronous time trace of the polariton intensity showing that the seemingly random jumps between the lower and upper states of the bistable system occur upon fluctuations getting larger than the threshold.

probabilities. Note that this excitation power corresponds to the power for which the hysteresis collapses with $0.14 \Delta \mathrm{B}$ noise strength. However, as the power is decreased to $0.20 \mathrm{~mW}$, or increased to $0.28 \mathrm{~mW}$, polaritons tend to become stable on the lower or on the upper state, respectively. We measure the time intervals spent in the lower (upper) branch before transition to the upper (lower) branch and construct a histogram of the number of counts as a function of time intervals. In Fig. 6 we show the histogram for time intervals where polaritons stay in the lower branch for the case of an input power of $0.24 \mathrm{~mW}$ and applied noise of $0.48 \Delta \mathrm{B}$. This histogram gives an exponential decay with a characteristic time, called the residence time, of $17.5 \mu$ s (inset of Fig. 6). Note that the residence time, the time period the system stays in each branch before it undergoes a transition, depends on both the laser power and noise strength. 


\section{EXPERIMENT}

(a)
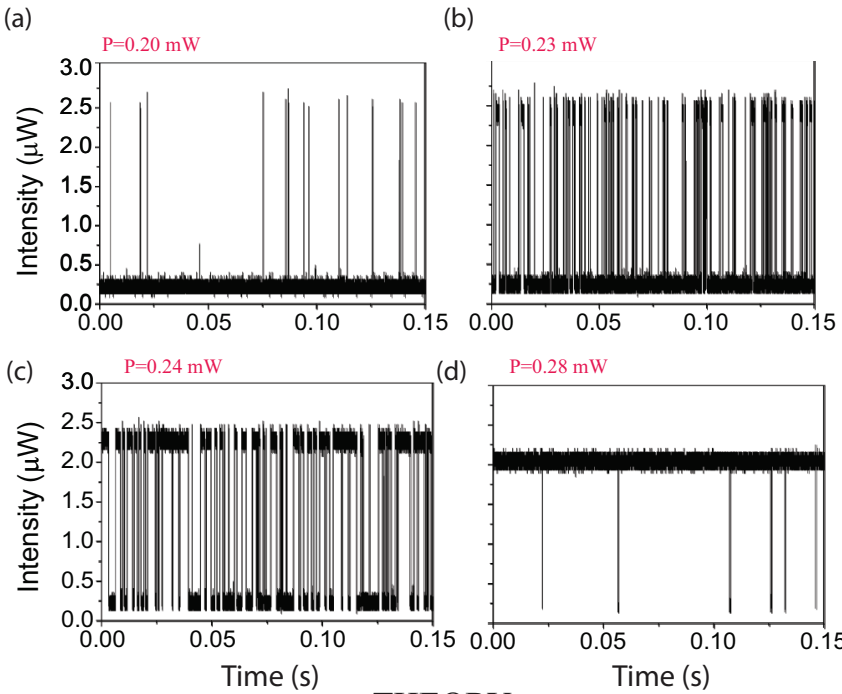

THEORY

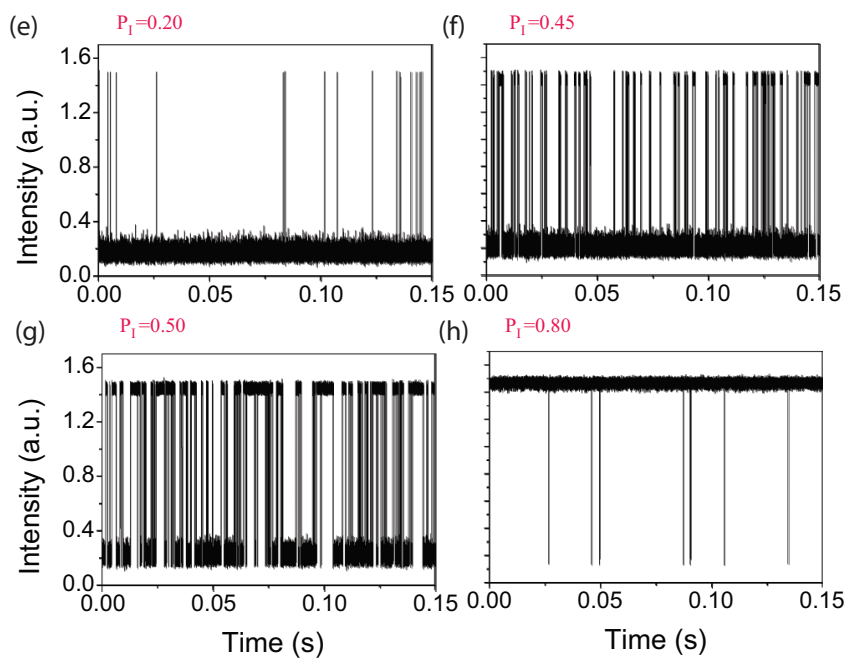

FIG. 5. (Color online) Typical time traces of the polariton transitions between the two stable states. (a),(b) For laser powers below the middle of the bistability loop $(P=0.20,0.23 \mathrm{~mW})$. Here the lower state is the more stable state. (c) For the laser power around the middle of bistability $(P=0.24 \mathrm{~mW})$, polariton transitions between the two stable states occur with the same probability. (d) For a laser power close to the upper threshold $(P=0.28 \mathrm{~mW})$, the upper branch is the most stable state and we only observe some random jumps to the lower state. In (a)-(d), the applied noise strength is $D=0.30$ $\Delta B$. (e)-(h) Theoretical realizations in the same conditions as for (a)-(d). They reproduce very accurately the observed behavior. The input powers are, respectively, $P_{I}=0.20$ in (e), 0.45 in (f), 0.5 in (g), and 0.8 in (h). In (e)-(h) the noise strength is $D=0.20 \Delta \mathrm{B}$.

In Figs. 7(a) and 7(b), we plot, for different input laser powers, the residence time in the lower and upper branches as a function of the noise strength. It appears that for both branches the residence times decrease as the noise strength increases. This is expected because, as we mentioned above, the input laser power fluctuations induce fluctuations in the polariton population, which produce instability in the hysteresis cycle. In the lower branch, the residence time decreases as the power is increased toward the upper threshold, and the reverse occurs

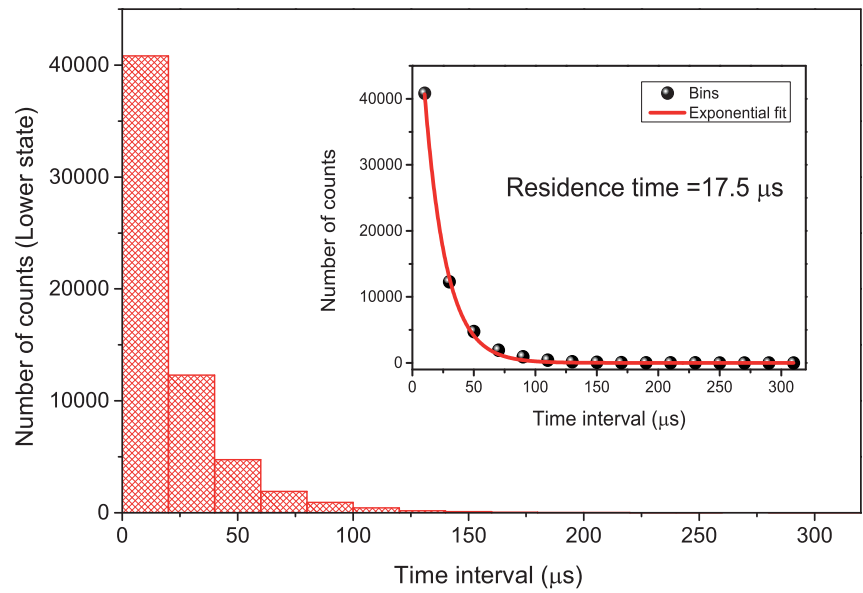

FIG. 6. (Color online) Residence time probability distribution. Histogram of the lower-state residence time for an input noise intensity of $D=0.48 \Delta \mathrm{B}$ at $P=0.24 \mathrm{~mW}$. (Inset) Histogram of the distribution showing the exponential decay of $17.5 \mu \mathrm{s}$.

in the upper branch. Note that, for $0.24 \mathrm{~mW}$ input power, the residence times for the lower and the upper branches coincide. This is the so-called Kramers time [24]. It has to be pointed out that $0.24 \mathrm{~mW}$ is the power at which the hysteresis cycle collapses for $0.14 \Delta \mathrm{B}$ noise (Fig. 2) and also corresponds to the middle power of the hysteresis loops measured for lower amounts of noise [Fig. 3(b)]. This result shows that, at this (a)

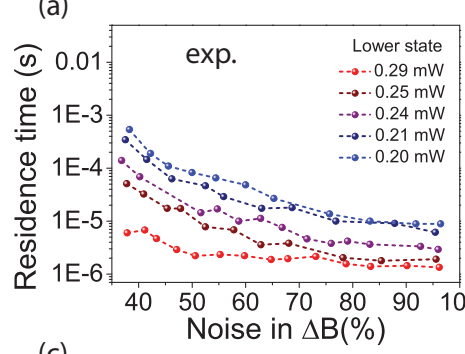

(b)

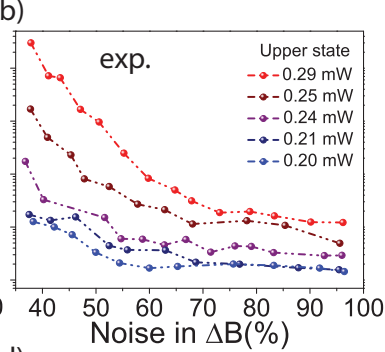

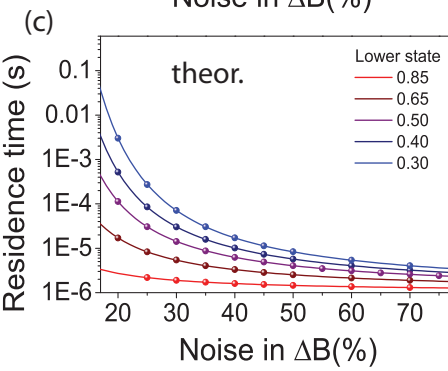

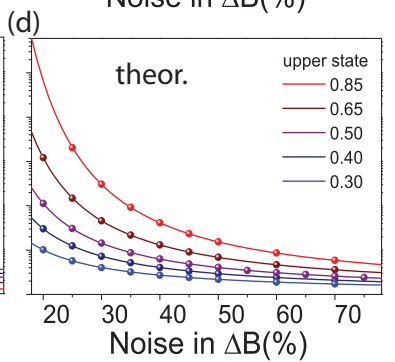

FIG. 7. (Color online) Polariton residence time for lower and upper states. (a) Residence time of the lower state for five different laser powers: $P=0.20,0.21,0.24,0.25$, and $0.29 \mathrm{~mW}$. The noise intensity is increased from $D=0.37$ to $0.96 \Delta \mathrm{B}$. (b) Residence time of the upper state for the same amounts of laser power and noise. At $P=0.24 \mathrm{~mW}$ residence times of the lower and the upper states are nearly the same. (c) Theoretical residence time of the lower state for five different laser powers: $P_{I}=0.30,0.40,0.50,0.65$, and 0.85 . The noise intensity is increased from $D=0.2$ to $1 \Delta \mathrm{B}$. (d) Theoretical residence time of the upper state for the same amounts of laser power and noise. Calculation from GPE (points) and theoretical model (line) are both presented. 
(a)

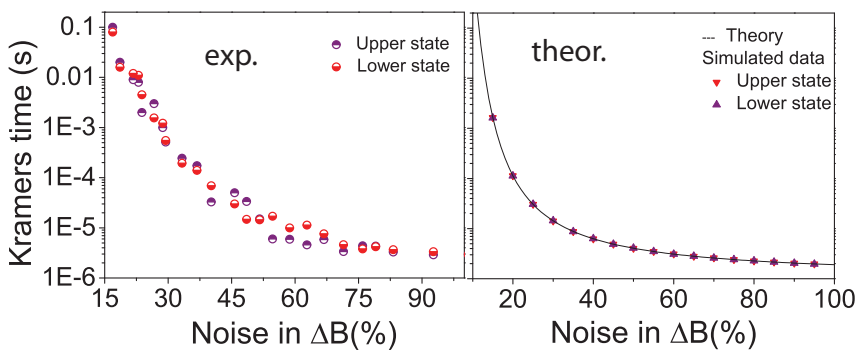

FIG. 8. (Color online) Polariton Kramers time. (a) Experimental polariton residence time for the lower (pink) and the upper (purple) states versus laser noise at the middle of polariton bistability $(P=$ $0.24 \mathrm{~mW}$ ). At this position, the two residence times are nearly equal, which are then labeled as the Kramers time. The noise intensity is tuned from $D=0.16$ to $0.93 \Delta \mathrm{B}$. When increasing the noise intensity, the Kramers time is observed to decrease. The minimum measured Kramers time is limited to the experimental bandwidth. (b) The theoretical residence times of the lower and the upper states for the laser power around the middle of bistability $\left(P_{I}=0.5\right)$, which is called Kramers time. Calculation from GPE (points) and theoretical statistical model (line) are both presented.

power, the polaritons have the same probability to stay in the lower and the upper bistable states.

We display in Fig. 8(a) the residence time, measured at a power of $0.24 \mathrm{~mW}$, in the upper and lower branch for a large variation of the noise strength. This result shows that both residence times coincide, showing that the probability to remain in the upper and lower branch is the same whatever is the strength of applied noise. It is worth mentioning that the minimum values measured for both residence times are determined by the noise correlation time $(1 \mu \mathrm{s})$.

We would like to comment on the fact that the applied noise acts as an effective source of internal noise on the polariton system. This is shown to affect the overall character of the bistable system. In the stochastic resonance phenomenon [28], which involves the counterintuitive concept that, under certain conditions, adding noise to a modulated bistable system actually increases its coherent response. Having established that the laser noise is a possible way of coupling external noise to the polariton system, it is indeed possible that, for the right amount of noise, one would see an increase in the coherent response of the amplified modulated signal, rather than the expected decrease. As a matter of fact, stochastic resonance has been observed in polariton bistable systems $[29,30]$. It has been shown that, by applying in the input laser power a small amplitude modulation with frequency around $1 \mathrm{kHz}$, the stochastic resonance appears for a range of noise power (between 0.1 to $0.3 \Delta \mathrm{B}$ ) in the polariton system [29]. Actually here we found that, for this amount of noise, the Kramers time is of the order of milliseconds, which is indeed around the modulation period, as expected for the observation of stochastic resonance [31]. Notice that the hysteresis cycle appears as a discriminator for this amount of noise [Fig. 2(a)]. This effective noise value fixes the limit for the applied modulation frequency for the observation of stochastic resonance.

\section{THEORETICAL MODEL}

The usual model to describe the polariton multistable systems is based on a Gross-Pitaevskii equation [32]. Here, in order to model the polariton dynamics in a noisy bistable regime, we consider two different approaches. First, we perform numerical simulation using the Gross-Pitaevskii equation (GPE) with a stochastic perturbation. Second, starting with the polariton bistability without any external noise, we consider the statistical behavior of the system.

For the numerical simulation we use the nonlinear GPE,

$$
i \frac{d \Psi}{d t}=-\Delta \Psi-i \gamma_{p} \Psi+\alpha_{1}|\Psi|^{2} \Psi+F,
$$

where $\Psi, \gamma_{p}$, and $\alpha_{1}$ are the polariton field, the polariton linewidth, and the interaction constant, respectively. $\Delta$ represents the energy detuning between the laser and the polariton ground-state energy. The driving field $F$ is defined as

$$
F(t)=\sqrt{I+D(t)},
$$

where $I$ is the laser intensity and $D$ is the random perturbation term which acts on the intensity. The perturbation is considered as a Gaussian noise with a standard deviation $\sigma$.

\section{A. Static regime}

In Figs. 2(c) and 2(d) we display the simulated polariton bistability without noise (black dashed line) to extract the theoretical width $\Delta \mathrm{B}$. We also display the bistabilities for noise with different standard deviation values normalized with respect to $\triangle \mathrm{B}$. The parameters used for the GPE to reproduce the experimental bistable behavior are $\gamma_{p}=0.08 \mathrm{meV}, \alpha_{1}=$ $0.34 \mathrm{meV}$, and $\Delta=0.4 \mathrm{meV}$. We observe a qualitative agreement between the experimental [Figs. 2(a) and 2(b)] and theoretical [Figs. 2(c) and 2(d)] results. It is worth mentioning that the threshold power between the lower and the upper state depends on the characteristic time of the experiment compared to the residence time for a given noise strength, which is taken into account by the number of iterations in the simulation.

In Figs. 3(c) and 3(d) we display the simulated hysteresis width and the input power corresponding to the middle position of the respective hysteresis loops as a function of the applied noise strength, respectively. As clearly seen, the numerical simulations reproduce really well the three characteristic behaviors of the polariton dynamics in a noisy bistability regime: (1) the reduction of the bistability width for increasing noise power, (2) the linear dependence of this reduction, and (3) the stability of the hysteresis middle point. It is important to note that theoretical bistability width, without laser noise, is larger than corresponding experimental $\Delta \mathrm{B}$. This discrepancy originates from mechanical or internal fluctuations which are present during the experimental measurements.

\section{B. Dynamic regime}

Figures 5(e) to 5(h) show the simulated output time streams for different input powers and at a fixed noise standard deviation $D=0.20 \Delta \mathrm{B}$. The noise strength and input powers in GPE are chosen to reproduce the experimental data. By using the same analysis procedure as for experimental data, we 
extract the residence time [Figs. 7(c) and 7(d)] and Kramers time [Fig. 8(b)].

To model the dynamics of the system between the lower and the upper states, we also use a statistical approach according to the hysteresis loop without noise. Considering that the noise affects only the intensity and not the phase of the driving field, both thresholds on the hysteresis loop are fixed and perfectly defined. We name these intensity thresholds $I_{\text {up }}$ when the polaritons pass from the lower state to the upper state and $I_{\text {down }}$ for the opposite transition.

In this context, the noise perturbation $D$ is modeled by a standard normal distribution with a characteristic correlation time $\left(\tau_{\text {cor }}\right)$ given by the bandwidth of the experimental components. For a given mean power $I_{0}$ the noise intensity distribution becomes

$$
N(I)=\frac{1}{\sigma \sqrt{2 \pi}} \exp \left[-\frac{1}{2}\left(\frac{I-I_{0}}{\sigma}\right)^{2}\right],
$$

where $I_{0}$ and $\sigma$ are defined as a function of the $\Delta \mathrm{B}=I_{\text {up }}-$ $I_{\text {down }}$,

$$
\begin{gathered}
I_{0}=I_{\text {down }}+P_{I} \Delta \mathrm{B}, \\
\sigma=P_{\sigma} \Delta \mathrm{B},
\end{gathered}
$$

where $P_{I}=0,0.5$, and 1 represent lower threshold, middle of optical bistability, and upper threshold, respectively. Finally, we can determine the conditional probability for the system to transit from the initial state by the cumulative distribution function (CDF). For example, when the polariton population is in the lower state, the probability to pass to the upper state is given by

$$
P\left(I>I_{\text {up }} \mid \text { down }\right)=\int_{I_{\mathrm{UP}}}^{\infty} I(x) d x=\frac{1}{2}\left[1-\operatorname{erf}\left(\frac{1-P_{I}}{P_{\sigma} \sqrt{2}}\right)\right],
$$

where $\operatorname{erf}(x)$ is the error function.

From a statistical point of view, the histogram built in Fig. 6 represents the non-normalized probability to jump at time $t$ knowing that the system was located on the lower state for $t$ seconds. This probability function can be written as an analytical form,

$$
W(n)=P_{s}^{(n-1)} P_{j},
$$

where $P_{j}$ is the probability to jump from initial state to the other stable state, and $P_{s}=1-P_{j}$ is the probability to stay in the initial state. $n$ represents the number of iterations. Equation (7) can be written as

$$
W(n)=\frac{P_{j}}{P_{s}} \exp \left[n \ln \left(P_{s}\right)\right] .
$$

Considering the noise correlation time $\left(\tau_{\text {cor }}\right)$ as the minimum time step, this equation can be rewritten as

$$
W(t)=\frac{P_{j}}{P_{s}} \exp \left[\frac{t}{\tau_{\text {cor }}} \ln \left(P_{s}\right)\right] .
$$

Similarly to the experimental procedure, we can extract the general residence time $\left(\tau_{\text {res }}\right)$,

$$
\tau_{\mathrm{res}}=-\frac{\tau_{\mathrm{cor}}}{\ln \left(P_{S}\right)}
$$

and the particular Kramers time when

$$
\begin{aligned}
P\left(I>I_{\text {up }} \mid \text { down }\right) & =P\left(I<I_{\text {down }} \mid \text { up }\right) \\
& =\frac{1}{2}\left[1+\operatorname{erf}\left(\frac{0.5}{P_{\sigma} \sqrt{2}}\right)\right] .
\end{aligned}
$$

Considering the statistical theory, we add in Figs. 7(c) and 7(d) and 8(b) the residence time and the Kramers time for different input laser powers as a function of the noise strength. The statistical description is in agreement both with the experiment and the GPE simulation. We can also extract certain experimental parameters such as the noise correlation time $\tau_{\text {cor }}=1 \mu \mathrm{s}$, which corresponds to the experimental value. All characteristic times of the system can be tuned precisely by varying the Gaussian noise correlation time, while the main mechanism would be the same. We would like to emphasize that there is a cutoff correlation time around the order of magnitude of the characteristic of the polariton dynamics at which the mentioned mechanism does not work anymore.

\section{CONCLUSION}

In conclusion, we have presented the noise dependence of the bistable properties of a polariton system. We have evidenced that the applied noise acts as an effective source of internal noise on the system. In the low-noise regime, the hysteresis is well defined and shows high-quality emission stability in each branch. We have shown that the upper (lower) threshold power decreases (increases) with noise strength, which induces polariton escape to the other branch. This results in reduction to the bistability width. We have demonstrated that a noise-strength threshold exists in which the bistability collapses and the system behaves as a discriminator. The results reveal that the threshold noise strength brings in loss of fidelity for devices. For larger amounts of noise we have determined the residence times for different input powers through time-resolved emission measurements. We have demonstrated that at collapsing power the probability of polaritons being in the upper and lower branches is the same whatever the strength of applied noise. In this particular situation, we have defined the Kramers time, which is measured as a function of a large range of applied noise. Through numerical simulations using GPE driven by stochastic excitation, we have reproduced the experimental results. The experimental procedure and the theoretical background exposed in the present paper might be extended to other bistable structures. This paper presents key elements to perform a study of noise-induced phenomena in nonlinear systems, a prerequisite for device performance evaluation.

\section{ACKNOWLEDGMENTS}

The present work has been supported by the Swiss National Science Foundation under Project No. N135003, the Quantum Photonics National Center of Competence in Research under Project No. N115509, and the European Research Council under Project POLARITONICS Contract No. N219120. The POLATOM Network is also acknowledged. 
[1] A. Szöke, V. Daneu, J. Goldhar, and N. Kurnit, Appl. Phys. Lett. 15, 376 (1969).

[2] L. A. Lugiato, P. Mandel, S. T. Dembinski, and A. Kossakowski, Phys. Rev. A 18, 238 (1978).

[3] E. Abraham and S. Smith, Rep. Prog. Phys. 45, 815 (1982).

[4] R. Roy and L. Mandel, Opt. Commun. 34, 133 (1980).

[5] E. Arimondo and B. Dinelli, Opt. Commun. 44, 277 (1983).

[6] G. P. Agrawal and N. K. Dutta, J. Appl. Phys. 56, 664 (1984).

[7] A. Joshi and M. Xiao, Phys. Rev. Lett. 91, 143904 (2003).

[8] A. Joshi, W. Yang, and M. Xiao, Phys. Lett. A 315, 203 (2003).

[9] B. Sfez, J. Oudar, J. Michel, R. Kuszelewicz, and R. Azoulay, Appl. Phys. Lett. 57, 324 (1990).

[10] S. H. Asadpour and H. R. Soleimani, Opt. Commun. 321, 104 (2014).

[11] H. Gibbs, S. McCall, T. Venkatesan, A. Gossard, A. Passner, and W. Wiegmann, Appl. Phys. Lett. 35, 451 (1979).

[12] A. Baas, J. P. Karr, H. Eleuch, and E. Giacobino, Phys. Rev. A 69, 023809 (2004).

[13] T. Paraïso, M. Wouters, Y. Léger, F. Mourier-Genoud, and B. Deveaud-Plédran, Nat. Mater. 10, 80 (2011).

[14] D. Miller, D. Chemla, T. Damen, A. Gossard, W. Wiegmann, T. Wood, and C. Burrus, Appl. Phys. Lett. 45, 13 (1984).

[15] H. Gibbs, Optical Bistability: Controlling Light with Light (Elsevier, Amsterdam, 2012).

[16] Y. Takase, P. T. Thanh, R. Fujimura, and K. Kajikawa, Appl. Phys. Express 7, 042202 (2014).

[17] A. Amo, T. Liew, C. Adrados, R. Houdré, E. Giacobino, A. Kavokin, and A. Bramati, Nat. Photon. 4, 361 (2010).

[18] R. Cerna, Y. Léger, T. K. Paraïso, M. Wouters, F. MorierGenoud, M. T. Portella-Oberli, and B. Deveaud, Nat. Commun. 4, 2008 (2013).
[19] D. Ballarini, M. De Giorgi, E. Cancellieri, R. Houdré, E. Giacobino, R. Cingolani, A. Bramati, G. Gigli, and D. Sanvitto, Nat. Commun. 4, 1778 (2013).

[20] G. Grosso, S. Trebaol, M. Wouters, F. Morier-Genoud, M. T. Portella-Oberli, and B. Deveaud, Phys. Rev. B 90, 045307 (2014).

[21] T. C. H. Liew, A. V. Kavokin, and I. A. Shelykh, Phys. Rev. Lett. 101, 016402 (2008).

[22] L. Gammaitoni, F. Marchesoni, E. Menichella-Saetta, and S. Santucci, Phys. Rev. Lett. 62, 349 (1989).

[23] R. Johne, N. S. Maslova, and N. A. Gippius, Solid State Commun. 149, 496 (2009).

[24] H. A. Kramers, Physica (Amsterdam) 7, 284 (1940).

[25] C. Weisbuch, M. Nishioka, A. Ishikawa, and Y. Arakawa, Phys. Rev. Lett. 69, 3314 (1992).

[26] R. I. Kaitouni, O. El Daï, A. Baas, M. Richard, T. Paraiso, P. Lugan, T. Guillet, F. Morier-Genoud, J. Ganiere, J. Staehli et al., Phys. Rev. B 74, 155311 (2006).

[27] N. Takemura, S. Trebaol, M. Wouters, M. T. Portella-Oberli, and B. Deveaud, Phys. Rev. B 90, 195307 (2014).

[28] R. L. Badzey and P. Mohanty, Nature (London) 437, 995 (2005).

[29] H. Abbaspour, S. Trebaol, F. Morier-Genoud, M. T. PortellaOberli, and B. Deveaud, Phys. Rev. Lett. 113, 057401 (2014).

[30] H. Abbaspour, S. Trebaol, F. Morier-Genoud, M. T. PortellaOberli, and B. Deveaud, Phys. Rev. B 91, 155307 (2015).

[31] T. Wellens, V. Shatokhin, and A. Buchleitner, Rep. Prog. Phys. 67, 45 (2004).

[32] N. A. Gippius, I. A. Shelykh, D. D. Solnyshkov, S. S. Gavrilov, Y. G. Rubo, A. V. Kavokin, S. G. Tikhodeev, and G. Malpuech, Phys. Rev. Lett. 98, 236401 (2007). 